\title{
Editorial: Smart Objects and Technologies for Social Good
}

\author{
Catia Prandi ${ }^{1,2}$ - Johann M. Marquez-Barja ${ }^{3,4}$ \\ Published online: 8 April 2021 \\ (C) The Author(s), under exclusive licence to Springer Science+Business Media, LLC, part of Springer Nature 2021
}

\section{Editorial}

Social good can be defined as something (such as a "good" or a service) that benefits the largest number of people in the largest possible way. In other words, social good implies a positive societal impact in areas including climate change (e.g., clean air, clean water), healthcare, education (e.g., literacy).

In this context, the pervasiveness of today's smart objects and technologies are creating unprecedented opportunities for social good. In fact, connectivity and innovation in technology have the potential to impact our lives, tackling some of the world's toughest challenges in term of climate impact and environmental sustainability, diversity and inclusion in education and healthcare, and communities at risk, to nominate a few. A clear example is represented by the Internet-of-things (IoT), that taking advantage of smart objects with sensing and connectivity capabilities, can help in transforming our world for the better. In fact, along with advances in data analytics, blockchain technology, and low-power wide-area network, IoT-enabled devices and sensors are being used to address social issues such as reducing air pollution, creating a clean environment, save energy and water, create smarter agriculture and food supply systems.

This special issue features seven selected high-quality papers in the fields of LoRa architecture for rural areas, routing protocols in MANET, IoT-enabled data visualization for sustainability and safety, deep learning for human activity recognition, serious games in the context of cognitive behavioural therapy, blockchain both considering the users' social impact and its usage in E-Health Environments.

Catia Prandi

catia.prandi2@unibo.it

1 Departments of Computer Science and Engineering, University of Bologna, Via Zamboni, 33, 40126 Bologna, BO, Italy

2 ITI - LARSyS, Caminho da Penteada, 9020-105 Funchal, Portugal

3 University of Antwerp, Sint-Pietersvliet 7, B-2000 Antwerpen, Belgium

4 IMEC, Kapeldreef 75, B-3001 Leuven, Belgium
The first article entitled "LADEA: a software infrastructure for audio delivery and analytics" describes LADEA, a generic software infrastructure for delivering audio messages and enabling audio analytics exploiting a LoRa architecture that can include generic external data sources using an MQTT-based interface. The study is framed in the context of remote locations, like rural areas, with the goal of employing LADEA to provide two basic services: a voice messaging system that allows users who cannot read or write to send voice notes, and an audio compression service to extract the main features from the audio input to use it for developing intelligent ML-based audio analytics.

The second article, entitled "Location Dynamic Tabu Routing Protocol for MANETS" proposes a dynamic routing protocol based on a tabu search approach, relying on local network knowledge and in-packet short-term memory to alleviate the local minima problem. The authors investigated the behavior of the analyzed routing protocols in a general MANET, considering a wide range of application scenarios which may include sensors, vehicles, drones, mobile users, etc. in smart homes, campuses, cities, farms, and industries. To validate the proposed approach, the study presents an experimental assessment, measuring protocol performance under different configurations and profiles, evidencing its benefits.

In the next article, entitled "On exploiting Data Visualization and IoT for increasing sustainability and safety in a Smart Campus", a study concerning the design and implementation of a testbed where IoT and Data Visualization have been exploited to increase the sustainability and safety of a Smart Campus is presented. In particular, the authors report on the overall system architecture and the interactive dashboard that has been designed to facilitate the management of the campus premises and the timetabling. As case studies, the authors focused on improving the campus sustainability (in terms of energy saving) and safety (considering the COVID-19 restrictions and regulations).

The fourth article entitled "Alternative Deep Learning Architectures for Feature-Level Fusion in Human Activity Recognition" proposes new deep learning architectures to fuse data provided by multiple sensors. In order to make the data fusion possible, the authors exploited convolution, dense, and concatenation layers. To validate the proposed approaches in 
the Human Activity Recognition domain, the Mobile HEALTH dataset, which consists of inertial data and electrocardiograms collected from different sensors worn by subjects, has been exploited. Finally, the authors discuss the obtained results showing that the proposed deep learning architectures are suitable and promising for data fusion in HAR.

The fifth article, "An engaging serious game aiming at awareness of therapy skills associated with social anxiety disorder" presents the design of a serious game, built using the Unity $3 \mathrm{D}$ engine and $\mathrm{C} \#$ programming language, aiming at engaging university students in raising their awareness of cognitive behavioral therapy (CBT) skills associated with social anxiety disorders. The study was based on three different iterations (study 1 , study 2 , and study 3 ), all included within a formative evaluation framework. The authors report on the positive findings in terms of focused attention, perceived usability, aesthetic appeal, being worthwhile, and narrative understanding.

Next, "Analysis of Witnesses in the Steem Blockchain" presents an investigation on the witness users on the blockchain Steem and their contribution to the social platform Steemit (the most important blockchain-based social networking site) and their social impact. To this end, the authors present two sets of analyses that cover the witnesses' blogs and their accounts. To anticipate here some results, the blog analyses show that witnesses are socially impactful users, they create lots of content, and the content they create are highly evaluated by the users in Steemit, while the accounts analyses confirm the social activity of witnesses and uncover additional details concerning their rewards, in particular as content creators and content curators.

To conclude the special issue, in the last article, entitled "Distributed Network Slicing Management Using Blockchains in E-Health Environments", the authors take advantage of the network slicing (NS) concept to create a solution for NPNs (e.g., hospital network) in e-health environments which maintains QoS and privacy requirements among slices. In addition, the authors propose a blockchain mechanism to secure the NS management layer. This mechanism ensures data integrity and reliability for the NSs settings. To assess their approach, the authors deployed a Proof of Concept using the 5G-EmPOWER system and a public blockchain, and they evaluated some performance metrics in different scenarios.

Acknowledgements The guest editors are thankful to our reviewers for their effort in reviewing the manuscripts. We also thank the Edit-in-Chief, Dr. Imrich, the Production Coordinators Leonora Panday and Jed Joseph Adel for their supportive guidance during the entire process.

Publisher's note Springer Nature remains neutral with regard to jurisdictional claims in published maps and institutional affiliations.

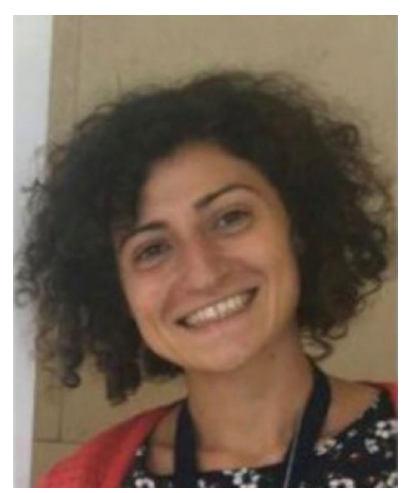

Catia Prandi, Ph.D, is an assistant professor at the Departments of Computer Science and Engineering at the University of Bologna (Italy). She is also a faculty member of the Interactive Technologies Institute (ITI/ LARSyS), Portugal, since 2017. Her research interests focus on Human-Smart Object Interaction in mobile contexts and sustainability, with a specific interest in co-design methodologies and users' participation strategies, taking advantage of crowdsourcing and collaborative approaches to engage communities, as witnessed by almost 90 publications both in international conferences and journals. She is involved in the organization of some IEEE and ACM International Conferences, and she was the General Co-Chair of the 6th EAI International Conference on Smart Objects and Technologies for Social Good (GoodTechs 2020). She collaborated with different research groups in different international projects, and she is the principal investigator for the University of Bologna in the H2020 FET SMARTLAGOON project

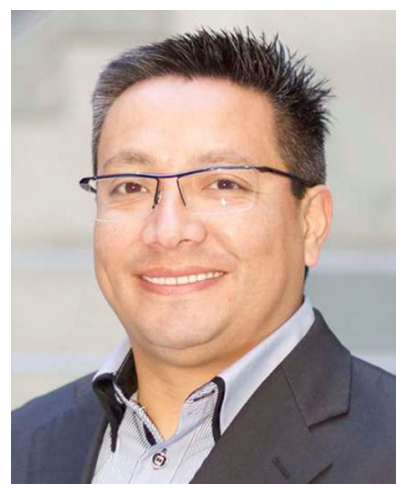

Johann M. Marquez-Barja , $\mathrm{Ph} . \mathrm{D}$, is a Professor at University of Antwerp, as well as a Professor in IMEC, Belgium. He is leading the Wireless Cluster at IDLab/ imec Antwerp. He was and is involved in several European research projects such as CREW, FORGE, WiSHFUL, Fed4FIRE/ FAVORITE, Fed4FIRE+, eWINE, CONCORDA, 5GCARMEN, FLEXNET, F U TEBOL ( Technical Coordinator), 5G-Mobix, PROTEGO, InterConnect, Dedicat-6G, Daemon, Vital-5G and 5G-Blueprint (Technical Coordinator) projects. He is a member of ACM, and a Senior member of the IEEE Communications Society, IEEE Vehicular Technology Society, and IEEE Education Society where he participates in the board of the Standards Committee. His main research interests are: $5 \mathrm{G}$ advanced architectures including edge computing; flexible and programmable future end-to-end networks; IoT communications and applications. He is also interested in vehicular communications, mobility, and smart cities deployments. Prof. Marquez-Barja is co-leading the Citylab Smart City testbed, part of the City of Things programme, and the SmartHighway testbed, both located in Antwerp, Belgium. Furthermore, he is also interested and active on education development, being heavily involved in different research actions to enhance engineering education, in particular remote experimentation in e-learning systems. Prof. Marquez-Barja has given several keynotes and invited talks in different major events, as well as received 30 awards in his career so far, and co-authored more than 140 articles. He is also serving as Editor and Guest editor for different International Journals, as well as participating in several Technical Programme and Organizing Committees for several worldwide conferences/congresses. 